\title{
Psicooncología
}

ISSN: $1696-7240$

\section{Mujeres Jóvenes con Cáncer de Mama: Necesidades de Apoyo en Atención y Resiliencia}

\author{
Carmen Lizette Gálvez-Hernández ${ }^{1, *}$; Andrea Ortega Mondragón; Cynthia Villarreal-Garza \\ Bertha Ramos del Río ${ }^{4}$
}

Recibido: 28 de marzo de 2018 / Aceptado: 20 de agosto de 2018

Resumen: Antecedentes: las mujeres menores de 40 años con cáncer de mama (CaMa) presentan características bio-psico-sociales únicas que ameritan ser atendidas y estudiadas. Objetivo: evaluar a) la percepción de necesidades de apoyo en la atención (NAT) insatisfechas y nivel de resiliencia, b) la diferencia de NAT entre pacientes en tratamiento y sobrevivientes y c) la relación entre NAT y resiliencia, y su asociación con variables clínicas y sociodemográficas, respectivamente. Método: se estudiaron transversalmente a 150 mujeres jóvenes con CaMa en tratamiento y sobrevivientes, con una encuesta de NAT y una de resiliencia mexicanas. Resultados: la mediana de edad de las participantes fue de 36 años. Las necesidades menos satisfechas fueron las de sistema de salud e información. El grupo de tratamiento presentó más NAT comparado con las sobrevivientes $(\mathrm{p}=0,005)$. Se encontró que cuanto más NAT insatisfechas (globales, psicológicas, cuidado y apoyo, e información), menores valores en los dominios de resiliencia $(\mathrm{p}<0,005)$ en ambos grupos y por separado. Conclusiones: las condiciones externas (infraestructura, servicios de salud proporcionados y apoyo en seguridad social), habilidades propias (auto-confianza, estructura y organización) y/o recursos de apoyo externo (social y familiar) pudieron contribuir a que las pacientes percibieran pocas NAT insatisfechas. Los resultados resaltan la relevancia clínica de implementar intervenciones basadas en resiliencia.

Palabras Clave: Cáncer de mama; mujeres jóvenes con cáncer de mama y sobrevivientes; necesidad de apoyo en la atención; resiliencia.

\section{[en] Young Women with Breast Cancer: Supportive Care Needs and Resilience}

Abstract: Background: Women with breast cancer (BC) under 40 years of age have unique and important bio-psycho-social characteristics that should be assessed. Objective: To evaluate: a) the unmet supportive care needs $(\mathrm{SCN})$ perception and the level of resilience, $b$ ) the difference in $\mathrm{SCN}$ between patients in active treatment and survivors, c) the relationship between $\mathrm{SCN}$ and resilience, in addition to its relationship

1 Carmen Lizette Gálvez-Hernández. Catedrática-CONACyT. Instituto Nacional de Cancerología. México. Joven y Fuerte: Programa para la Atención e Investigación de Mujeres Jóvenes con Cáncer de Mama, Ciudad de México. México. E-mail:lizettegalvezh@gmail.com

2 Andrea Ortega Mondragón. Facultad de Estudios Superiores Zaragoza, Universidad Nacional Autónoma de México. México

E-mail:1andy.om@gmail.com

3 Cynthia Villarreal-Garza. Centro de Cáncer de Mama del Hospital Zambrano Hellion, Tecnológico de Monterrey, San Pedro Garza García, Nuevo León. México.

E-mail:cynthiavg@gmail.com

4 Bertha Ramos del Río. Facultad de Estudios Superiores Zaragoza, Universidad Nacional Autónoma de México. México. E-mail:becaau@yahoo.com

* Dirección de correspondencia: Carmen Lizette Gálvez-Hernández. Instituto Nacional de Cancerología. Av. San Fernando No. 22. Col. Sección XVI. C.P. 14080. Ciudad de México. Email: lizettegalvezh@gmail.com 
with clinical, and sociodemographic variables, respectively. Method: The unmet SCN and the level of resilience were cross-sectionally assessed in 150 young women with BC. Results: Participants had a median age of 36 years. The highest unmet needs were related to the healthcare system and information needs. The most unmet needs were reported by the group undergoing treatment compared to those in surveillance $(\mathrm{p}=.005)$. We found a significant and negative relationship between unmet SCN (global, psychological, care and support, and information) and resilience domains $(\mathrm{P}<.005)$, in both groups and separately. Conclusions: The perception of low unmet needs could be caused by external conditions (such as infrastructure, health services provided and social security support), abilities' perception (such as self-confidence, structure and organization) and/or support resources perceived (social and family). These results highlight the clinical relevance to address interventions based on resilience.

Keywords: Breast Cancer; young women with breast cancer; survivors; unmet needs; supportive care needs; resilience.

Sumario. 1. Introducción 2. Método 2.1. Muestra 2.2. Instrumentos 2.3. Procedimiento 2.4. Análisis de datos 3. Resultados 3.1. Necesidades de Apoyo en la Atención (NAT) 3.2. Resiliencia 3.3. Relación entre NAT y resiliencia 3.4. Variables sociodemográficas y clínicas, NAT y resiliencia 4. Discusión 5. Conclusiones 6. Referencias bibliográficas.

Cómo citar: Gálvez-Hernández CL, Ortega Mondragón A, Villarreal-Garza C, Ramos del Río B. Mujeres Jóvenes con Cáncer de Mama: Necesidades de Apoyo en Atención y Resiliencia. Psicooncología 2018;15:287-300. doi: 10.5209/PSIC.61436.

\section{Introducción}

La incidencia y la mortalidad por cáncer de mama (CaMa) en mujeres menores de 40 años en México y Latinoamérica corresponden a una gran proporción del total de pacientes ${ }^{(1-3)}$. Estas mujeres representan un grupo único con características biopsicosociales específicas, en comparación con mujeres de mayor edad ${ }^{(4,5)}$. Es decir, presentan tumores más agresivos al momento del diagnóstico -tumores triples negativo, sobreexposición de HER2, luminales $\mathrm{B}^{(6,7)}$ y mayor riesgo de recurrencia de la enfermedad en cualquier estadio clínico ${ }^{(8)}$. Además, presentan intereses y/o preocupaciones propias de la etapa de vida en la que se encuentran -fertilidad, autoimagen, calidad de vida, objetivos personales, entre otros ${ }^{(3,4,9)}$.

Se sabe que estas características generan necesidades de apoyo en la atención (NAT) ${ }^{(10)}$, que pueden no estar adecuadamente abordadas tanto a nivel individual, como dentro de los programas de capacitación oncológica ${ }^{(11)}$. Particularmente, los países desarrollados que exploran las NAT han descrito que las mujeres jóvenes con $\mathrm{CaMa}$ en tratamiento $\mathrm{y} / \mathrm{o}$ en fase de supervivencia informan tener necesidades: a) físicas, normalmente relacionadas a los efectos secundarios de los tratamientos, como síntomas menopaúsicos, problemáticas de fertilidad y afección en su sexualidad; b) necesidades psicológicas y emocionales, como apoyo en el manejo de ansiedad y depresión, miedo a la recurrencia, autoimagen, conexión con otras mujeres jóvenes; c) necesidades prácticas, como soporte con el impacto del cáncer en la vida diaria, inestabilidad en el trabajo, problemas financieros, cuidado de hijos, apoyo después del tratamiento; y d) necesidades de sistema de salud e información (SSI) con respecto al diagnóstico, planes de tratamiento, acceso a servicios, toma de decisiones ${ }^{(12-15)}$.

Por otro lado, debido al estrés y el riesgo que implica el diagnóstico de CaMa para la salud física y mental ${ }^{(16)}$, se ha considerado estudiar el rol de variables que promueven potencialidades, capacidades, talentos y recursos propios en pacientes oncológicos ${ }^{(17)}$. La resiliencia, definida como la capacidad de las personas de sobreponerse a periodos de 
dolor emocional y situaciones adversas ${ }^{(18)}$, se ha asociado positivamente con bienestar emocional, salud física, mental y calidad de vida en mujeres con CaMa mayores de 40 años y/o sobrevivientes; además, es un factor protector para la ansiedad, depresión y estrés ${ }^{(19-21)}$. Inclusive, se ha demostrado en pacientes oncológicos con distintos tipos de cáncer, que aquellos con niveles altos de resiliencia presentan menos $\mathrm{NAT}^{(22,23)}$.

No obstante, hasta el momento no se tiene conocimiento preciso acerca de las NAT y de los niveles de resiliencia en pacientes jóvenes mexicanas con CaMa, ni de la relación entre estas variables. De igual forma, se conoce poco si la etapa de tratamiento en la que se encuentran pudiera influir en los niveles de NAT ${ }^{(21,24-26)}$. Esta investigación está fundamentada en la importancia de identificar problemáticas sensibles a diferencias culturales, como las NAT, en relación con la exploración de recursos psicológicos de pacientes con CaMa expuestas a condiciones biopsicosociales particulares, lo que eventualmente permitirá el desarrollo y la implementación de intervenciones terapéuticas basadas en evidencia, dirigidas a estas pacientes.

Los objetivos de esta investigación fueron evaluar en mujeres jóvenes con CaMa: (1) la percepción de NAT insatisfechas y el nivel de resiliencia, (2) las diferencias en puntuaciones de NAT y resiliencia entre pacientes en tratamiento y en sobrevivencia, (3) la relación entre NAT y resiliencia, y (4) la relación de NAT y resiliencia con variables clínicas y sociodemográficas.

\section{Método}

\subsection{Muestra}

Participaron 150 mujeres de 18 a 40 años de edad del Depto. de Tumores Mamarios del Instituto Nacional de Cancerología, en la Ciudad de México. Todas con diagnóstico confirmado de CaMa, que estuvieran bajo tratamiento médico oncológico o fueran sobrevivientes en vigilancia (aquellas pacientes que habían concluido con el tratamiento oncológico según el expediente clínico) y supieran leer y escribir. Para la muestra, se excluyó a las pacientes que presentaran recurrencia de la enfermedad, se encontraran bajo tratamiento paliativo o expresaran su deseo de no participar.

\subsection{Instrumentos}

Encuesta de Necesidades de Apoyo en la Atención. Forma Corta 32 Versión Mexicana (SCNS-SF32 M) ${ }^{(27)}$ validada para población mexicana con CaMa por Gálvez et al. ${ }^{(28)}$. Esta escala evalúa las necesidades experimentadas durante el último mes como resultado de tener cáncer, así como su magnitud. Consta de 32 reactivos, respondidos en escala Likert, y está dividida en tres dominios: 1) SSI, 2) psicológicas, y 3) físicas y de la vida diaria; y dos indicadores: 1) sexualidad y 2) cuidados y apoyo al paciente. La versión mexicana dio cuenta del $60 \%$ de la varianza y su confiabilidad en consistencia interna fue de 0,936 .

Escala de Resiliencia Mexicana (RESI-M) ${ }^{(29)}$ evalúa habilidades que ayudan a los individuos a enfrentar la vida. Consta de 43 reactivos respondidos en escala Likert, divididos en 5 dominios: 1) fortaleza y confianza en sí mismo, 2) competencia social, 3) apoyo familiar, 4) apoyo social y 5) estructura. El análisis de validez dio cuenta del $43.60 \%$ de la varianza total del instrumento y su confiabilidad en consistencia interna fue de 0,93 . 


\subsection{Procedimiento}

El proyecto fue aprobado en sus aspectos éticos y de calidad de investigación por el Comité de Ética e Investigación del Instituto Nacional de Cancerología (015/018/ IBI) (CEI/968/15).

Se identificó anticipadamente a las pacientes que cumplían con los criterios de inclusión para participar en el estudio. Posteriormente, fueron contactadas e invitadas a participar. Aquellas que aceptaron, firmaron el consentimiento informado de participación voluntaria (que garantizaba anonimato y confidencialidad de la información otorgada). Finalmente, se llevó a cabo la aplicación de los instrumentos en una sola ocasión. Debido al nivel de alfabetización de las pacientes ${ }^{(30)}$, los cuestionarios fueron administrados de manera oral por asistentes de investigación capacitados y experimentados, con la finalidad de maximizar la estandarización.

\subsection{Análisis de datos}

Al comprobar que los datos no cumplían con los criterios de normalidad y homocedasticidad (prueba de Kolmogorov-Smirnov; prueba de Levene) se optó por analizar las diferencias en cuanto a variables nominales sociodemográficas a través de chi cuadrada; mientras que para las variables de razón se utilizó prueba de MannWhitney. Para el análisis de las diferencias entre los grupos de NAT y resiliencia, se empleó la prueba Mann-Whitney. El estudio de las relaciones entre las variables se llevó a cabo mediante correlación de Spearman. El análisis de los datos se realizó con el programa estadístico Statistical Package for Social Sciences (SPSS 24) para Windows.

\section{Resultados}

La muestra consistió en 150 pacientes, quienes se dividieron en dos grupos (en tratamiento y sobrevivientes) de acuerdo con sus características clínicas y sociodemográficas. Las participantes tenían una mediana de edad de 36 años (intervalo de 23 a 40 años) y, aunque se encontró que las sobrevivientes $(\mathrm{Me}=37$ ) fueron significativamente mayores en edad que las de tratamiento $(\mathrm{Me}=35)$, todas eran menores de 40 años. En cuanto a escolaridad, 30\% de todas las pacientes contaba con estudios de secundaria, mientras que el $28 \%$ con estudios de licenciatura. En ambos grupos, el mayor porcentaje reportó ser casada (54\%); $51 \%$ fue ama de casa. El grupo en tratamiento tuvo significativamente menor tiempo de haber sido diagnosticado ( $M=7.5 ; D t=4,82$ meses $)$, que el de sobrevivientes $(M=36,8 ; D t=28,6$ meses) Véase tabla 1.

Aproximadamente el $40 \%$ de las pacientes de cada grupo se encontraba en etapas clínicas II - III. Se incluyeron 3 participantes en estadio IV, debido a que se encontraban en tratamiento activo, no paliativo (según el expediente clínico). Significativamente más mujeres del grupo de sobrevivientes (en comparación con las de tratamiento) habían recibido un tratamiento oncológico integral $(\mathrm{p}<0,0001)$. Se destaca que cirugía y quimioterapia fueron los tratamientos que más recibieron ( $78 \%$ y $76 \%$, respectivamente), considerando al total de pacientes (véase Tabla 1). 
Tabla 1. Características sociodemográficas y clínicas de la muestra

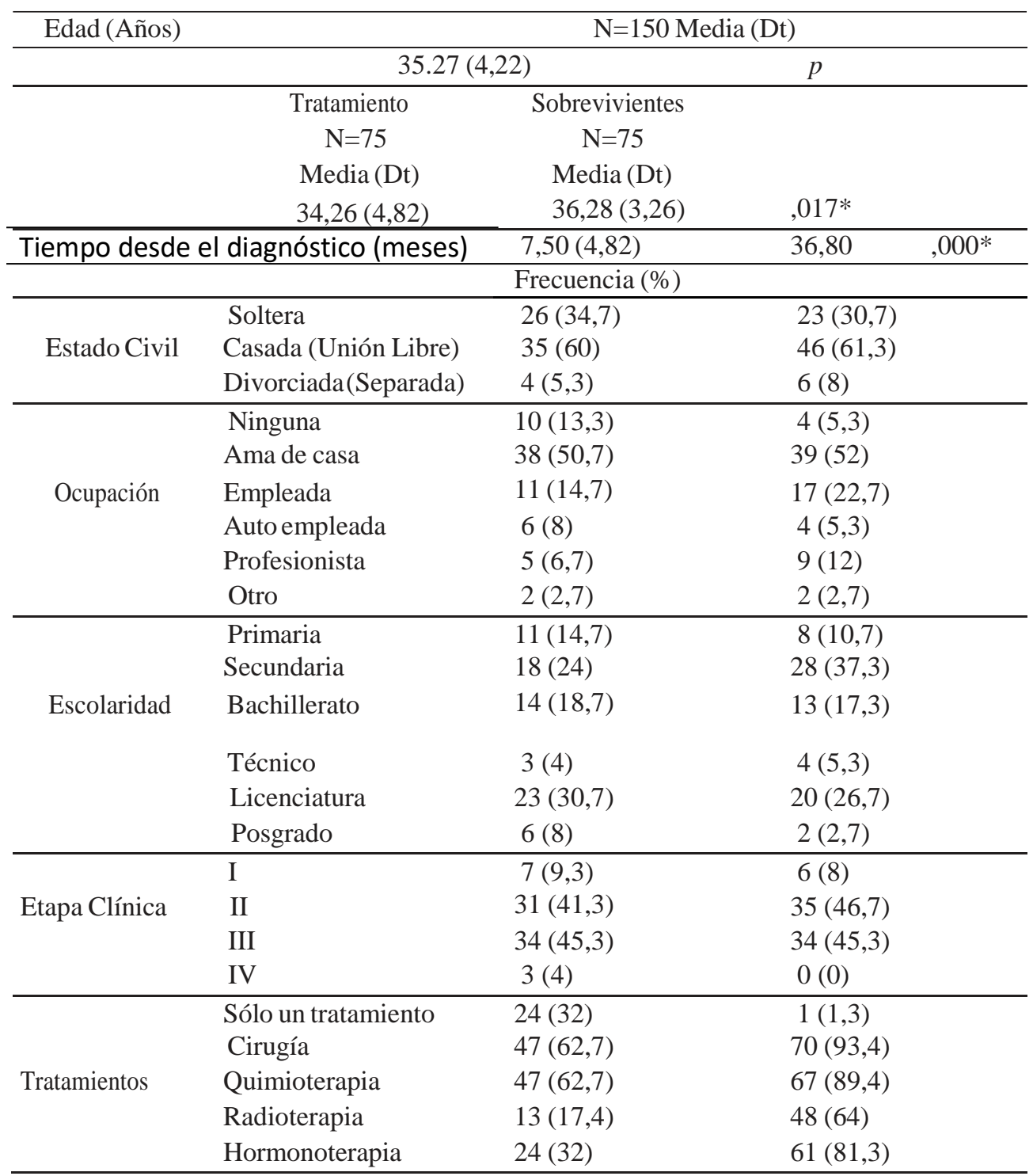

Nota: * significación estadística se tomaron en cuenta más de 1 respuesta

\subsection{Necesidades de Apoyo en la Atención (NAT)}

Todas las pacientes de la muestra reportaron baja percepción de NAT insatisfechas. La mediana del puntaje (estandarizado) en SCNS-SF32-M fue de 24,60 (0-100). El mayor porcentaje de necesidades insatisfechas se encontró en el dominio referente a $\mathrm{SSI}(\mathrm{Me}=26)$. El reactivo de mayor necesidad de toda la escala correspondió a "Estar informado acerca de que el cáncer está bajo control o disminuyendo".

Al comparar los grupos (Tabla 2), se observó que las mujeres en tratamiento presentaron significativamente mayor percepción global de NAT insatisfechas $(\mathrm{z}=-$ 
$2,83 ; \mathrm{p}=0,005)$, necesidades psicológicas $(\mathrm{z}=-2,20 ; \mathrm{p}=0,028)$, de SSI $(\mathrm{z}=-2,40$; $\mathrm{p}=0,016)$ y físicas $(\mathrm{z}=-2,80 ; \mathrm{p}=0,005)$. Ambos grupos mostraron niveles similares de NAT insatisfechas en necesidades del cuidado del paciente y sexuales.

El reactivo de mayor necesidad para el grupo en tratamiento fue "Necesidad de estar informado acerca de que el cáncer está bajo control o disminuyendo"; mientras que para las sobrevivientes fue "Miedo de que el cáncer se extienda o regrese". Estos reactivos pertenecen a los dominios de SSI y psicológicas, respectivamente.

Tabla 2. Diferencias entre mujeres en tratamiento y sobrevivientes en NAT

\begin{tabular}{lcccc}
\hline \multicolumn{1}{c}{ NAT } & \multicolumn{4}{c}{ Percepción de NAT insatisfechas } \\
\hline & $\begin{array}{c}\text { Tratamiento } \\
(\mathbf{N = 7 5 )}\end{array}$ & $\begin{array}{c}\text { Sobrevivientes } \\
(\mathbf{N = 7 5 )}\end{array}$ & \\
\cline { 2 - 5 } & \multicolumn{2}{c}{ Rango promedio } & $\mathbf{Z}$ & Sig. \\
\hline Global & 85,55 & 65,45 & $-2,83$ &, $005^{*}$ \\
Psicológicas & 83,30 & 67,00 & $-2,20$ &, $028^{*}$ \\
SSI & 84 & 67 & $-2,40$ &, $016^{*}$ \\
Físicas & 85,43 & 65,57 & $-2,80$ &, $005^{*}$ \\
Cuidados del paciente & 76,95 & $74,05)$ &,- 497 &, 620 \\
Sexuales & 78,73 & 72,27 & $-1,06$ &, 288 \\
\hline
\end{tabular}

Nota: *Significancia estadística

\subsection{Resiliencia}

La mediana del puntaje de resiliencia (estandarizado) en RESI-M de toda la muestra fue de 70,54 (48,84-100). Presentaron altos puntajes en todos los dominios de la escala, principalmente en apoyo social $(\mathrm{Me}=80)$ y familiar $(\mathrm{Me}=72,22)$.

Aunque el grupo de tratamiento obtuvo puntajes más bajos en todos los dominios de resiliencia (global, fortaleza, apoyo familiar y social, estructura) en comparación con las mujeres sobrevivientes, sólo se observaron diferencias significativas en competencia social $(\mathrm{z}=-2,86 ; \mathrm{p}=0,004)$. Véase Tabla 3 .

Tabla 3. Diferencias entre mujeres en tratamiento y sobrevivientes en RESI-M

\begin{tabular}{lcccc}
\hline \multicolumn{1}{c}{ RESI-M } & $\begin{array}{c}\text { Tratamiento } \\
(\mathbf{N = 7 5 )} \\
\text { Rango promedio }\end{array}$ & $\begin{array}{c}\text { Sobrevivientes } \\
(\mathbf{N = 7 5 )}\end{array}$ & $\mathbf{Z}$ & Sig. \\
\hline Global & 70,98 & 80,02 & $-1,275$ &, 202 \\
Fortaleza y confianza en sí mismo & 72,19 & 78,81 &,- 935 &, 350 \\
Competencia Social & 65,43 & 85,57 & $-2,86$ &, $004 *$ \\
Apoyo familiar & 75,59 & 75,41 &,- 027 &, 979 \\
Apoyo social & 77,91 & 73,09 &,- 723 &, 469 \\
Estructura & 70,49 & 80,51 & $-1,43$ &, 153 \\
\hline
\end{tabular}

Nota: * Significancia estadística 


\subsection{Relación entre NAT y resiliencia}

Considerando a toda la muestra en la Tabla 4, se encontró que entre más NAT global, menor puntaje en estructura -dominio de resiliencia- $(r=-0,261 ; p=0,001)$. En el grupo de tratamiento, entre más necesidades psicológicas menor, precepción de fortaleza y confianza en sí misma $(\mathrm{r}=-0,271 ; \mathrm{p}=0,009)$.

Tabla 4. Correlación entre los factores de NAT, Resiliencia y variables clínicas y sociodemográficas.

\begin{tabular}{|c|c|c|c|c|c|c|}
\hline & $\begin{array}{c}\text { Resiliencia } \\
\text { Global }\end{array}$ & $\begin{array}{c}\text { Fortaleza y } \\
\text { confianza }\end{array}$ & $\begin{array}{c}\text { Competencia } \\
\text { social }\end{array}$ & $\begin{array}{c}\text { Apoyo } \\
\text { familiar }\end{array}$ & $\begin{array}{l}\text { Apoyo } \\
\text { Social }\end{array}$ & Estructura \\
\hline \multicolumn{7}{|c|}{$\rho$ (Sig,) } \\
\hline \multicolumn{7}{|c|}{ Toda la muestra } \\
\hline Escolaridad & & ,275 $(, 000)$ & & & & \\
\hline Tiempo & & & ,263 (,001) & & & \\
\hline NAT global & & & & & &,$- 261(, 001)$ \\
\hline
\end{tabular}

\begin{tabular}{|c|c|c|c|c|c|}
\hline \multicolumn{6}{|c|}{ Tratamiento } \\
\hline Escolaridad & ,303 $(, 004)$ & ,304 (,004) & & & \\
\hline Edo, Civil & & &,$- 324(, 002)$ &,$- 294(, 005)$ & \\
\hline N, Psicológicas & &,$- 271(, 009)$ & & & \\
\hline \multicolumn{6}{|c|}{ Sobrevivientes } \\
\hline Escolaridad & & & &,$- 324(, 002)$ & \\
\hline NAT global & & & & &,$- 329(, 002)$ \\
\hline $\begin{array}{l}\text { N, Cuidado y } \\
\text { apoyo }\end{array}$ & $-, 361 * *, 001$ & $-, 404 * *, 000$ & $-, 313 * *, 003$ & $-, 287 * *, 006$ &,$- 388(, 000)$ \\
\hline $\begin{array}{l}\mathrm{N}, \text { Salud e } \\
\text { información }\end{array}$ & & & & &,$- 267(, 010)$ \\
\hline
\end{tabular}

$\mathrm{N}$ : necesidades

Enel grupo de las sobrevivientes, se encontró que a mayores NAT global insatisfechas, menor puntaje en estructura $(\mathrm{r}=-0,329 ; \mathrm{p}=0,002)$. Además, a mayores necesidades de cuidado y apoyo, menor puntaje en resiliencia global $(\mathrm{r}=-0,361 ; \mathrm{p}=0,001)$, fortaleza y confianza $(\mathrm{r}=-0,404 ; \mathrm{p}=0,000)$, apoyo familiar $(\mathrm{r}=-0,313 ; \mathrm{p}=0,003)$ y social $(\mathrm{r}=-0,287$; $\mathrm{p}=0,006)$, y en estructura $(\mathrm{r}=-0,388 ; \mathrm{p}<0,0001)$. Estructura también correlacionó negativamente con necesidades SSI $(\mathrm{r}=-0,267, \mathrm{p}=0,010)$.

\subsection{Variables sociodemográficas y clínicas, NAT y resiliencia}

Para la muestra completa de pacientes en la Tabla 4, se encontró que conforme el nivel de escolaridad aumenta, también lo hace la percepción de fortaleza y confianza $(\mathrm{r}=0,275 ; \mathrm{p}=<0,001)$. Inclusive, se identificó que a mayor tiempo desde el diagnóstico, mayor percepción de competencia social $(\mathrm{r}=0,272 ; \mathrm{p}=0,018)$. 
Con las pacientes en tratamiento se encontró una relación similar (como con toda la muestra) entre escolaridad y fortaleza y confianza $(\mathrm{r}=0,304 ; \mathrm{p}=0,004)$; así como entre escolaridad y puntaje global de resiliencia $(r=0,303 ; p=0,004)$. En este mismo grupo, se identificó una relación entre estado civil y apoyo familiar $(\mathrm{r}=-$ $0,324 ; \mathrm{p}=0,002)$ y social $(\mathrm{r}=0,294 ; \mathrm{p}=0,005)$.

En el grupo de las pacientes sobrevivientes se encontró que, entre mayor escolaridad, mayor puntaje en el dominio de apoyo social $(r=0,324 ; p=0,000)$.

\section{Discusión}

El objetivo del presente estudio fue evaluar una muestra de mujeres jóvenes con CaMa en tratamiento y sobrevivientes atendidas en un hospital de tercer nivel de referencia en la Ciudad de México. Dentro de los resultados, se encontraron niveles de NAT bajos en general, mientras que los de resilencia fueron altos. Las necesidades reportadas como más insatisfechas fueron las de SSI. El grupo en tratamiento fue el que más NAT insatisfechas reportó. En el de sobrevivientes, "el miedo a que el cáncer se extienda" fue la mayor NAT insatisfecha.

La relación entre NAT y resiliencia estuvo presente entre sus dominios, en toda la muestra, y en ambos grupos por separado (en tratamiento y sobrevivientes). Se encontró que, entre más necesidades insatisfechas, menores valores de resiliencia. Específicamente en las sobrevivientes, las necesidades de cuidado y apoyo se relacionaron con menor fortaleza y confianza, resiliencia global, apoyo familiar y social. Estructura se relacionó con NAT global (todas las pacientes), cuidado y apoyo y SSI (sobrevivientes). En el grupo en tratamiento, se encontró que las necesidades psicológicas insatisfechas estaban relacionadas negativamente con fortaleza y confianza.

No hubo relación entre NAT y variables sociodemográficas o clínicas. Se encontró que, a mayor escolaridad, mayor claridad en los objetivos, esfuerzo por alcanzar sus metas y auto-confianza (todas las pacientes y en tratamiento), resiliencia global (en tratamiento) y apoyo social en momentos difíciles (sobrevivientes). En todas las pacientes, a mayor tiempo desde el diagnóstico, mayor percepción de competencia para relacionarse con los demás y hacer nuevos amigos. El estado civil se asoció con apoyo familiar y social en las pacientes en tratamiento.

Los bajos niveles de percepción de NAT resultaron inconsistentes con respecto a estudios previos, que han reportado altos niveles ${ }^{(13-15,31,32)}$. Estos resultados pueden estar influidos por que la muestra fue recolectada en un centro de atención que ofrece manejo integral de la enfermedad, cobertura gratuita del tratamiento oncológico (para personas que carecen de seguridad social), oportunidad de participar en protocolos de investigación estandarizados a nivel internacional donde incorporan terapias innovadoras y, finalmente, cirugías para la reconstrucción mamaria como parte de la póliza del seguro ${ }^{(33)}$. Estos motivos pudieran influir en la satisfacción de necesidades percibidas.

La insatisfacción de las necesidades de SSI coincide con estudios previos realizados en mujeres jóvenes, que han referido necesidad de entender sus diagnósticos y planes de tratamiento, procedimientos, reportes patológicos y retrasos en el diagnóstico ${ }^{(13-15,31,32)}$. Este resultado pudiera explicarse por información poco específica, falta de claridad o uso de terminología complicada o técnica en las res- 
puestas otorgadas por el personal sanitario a las pacientes, así como el desconocimiento de las preferencias y peticiones de información que tienen las mismas ${ }^{(34-36)}$.

El reactivo con mayor necesidad está relacionado con información de que el cáncer esté bajo control, lo que contrasta con lo encontrado por Avis et al. ${ }^{(31)}$, quienes informan como necesidad principal "las preocupaciones por la menopausia prematura y problemas relacionados con el embarazo"; y con lo referido por Pedersen et al. ${ }^{(13)}$ y Rudy et al. ${ }^{(14)}$, quienes reportan como alta necesidad la fertilidad y la comunicación con los hijos. Estas discrepancias pudieran explicarse a través de las diferencias en las herramientas de evaluación, ya que dos de los estudios utilizaron instrumentos de evaluación distintos al presente, que evalúa directamente las necesidades con base en un modelo teórico, lo que limita las necesidades a explorar. Además, podría deberse a algunas características sociodemográficas - que no fueron evaluadas en este estudio- como el estado civil o el número de hijos, ya que éstas pueden definir la percepción de las NAT. En este sentido, algunos autores $^{(12,37)}$ mencionan que para las mujeres jóvenes con CaMa lo principal es satisfacer sus necesidades personales y asegurarse de recibir tratamientos adecuados como medida de protección, que les permita tomar decisiones y estar disponibles en un futuro para su familia o para alcanzar metas personales.

Los resultados de las NAT en tratamiento no fueron consistentes con la literatura antecedente $^{(12-15,31,32)}$. Esto puede deberse a que se trataba de estudios exploratorios que reclutaron pacientes con periodos largos de haber sido diagnosticadas (menos de 3 a 4 años), donde se les preguntaba si hubo necesidad en algún momento de la enfermedad. Dichos hallazgos no permiten precisar cuándo existió mayor necesidad o cuánto tiempo duró. Los resultados de este estudio identifican que, durante el tratamiento, la mayor necesidad es de SSI. Adicionalmente, en otro estudio refirieron que las necesidades durante el tratamiento fueron acerca de los efectos secundarios, fertilidad, menopausia y relaciones románticas después del cáncer ${ }^{(15)}$.

El reactivo de mayor necesidad de las sobrevivientes concuerda con Connel et al. ${ }^{(32)}$, quienes describieron que el miedo a la recurrencia y la incertidumbre hacia el futuro fue la mayor necesidad. Fitch ${ }^{(10)}$ propone que en la fase de sobrevivencia se presentan mayores necesidades debido a que las pacientes no se sienten adecuadamente informadas acerca de los efectos a largo plazo de la enfermedad y sobre cómo diferenciar síntomas de recurrencia; además de percibir pérdida de apoyo por parte del personal sanitario, una vez concluido el tratamiento. Adicionalmente, Ruddy et al. ${ }^{(14)}$ identificaron que estas pacientes requieren mayores conexiones con mujeres pares, mayor información del cuidado en la supervivencia, cómo manejar la fatiga, los problemas de memoria e imagen corporal.

La correlación negativa encontrada entre las variables NAT y resiliencia coincide y sostiene los resultados obtenidos por estudios antecedentes realizados con pacientes con algún diagnóstico de cáncer ${ }^{(22,23)}$. Particularmente, esta relación se presentó en mayor medida en las sobrevivientes, donde al percibir menor fortaleza y confianza propia, menor apoyo familiar y social refirieron mayor necesidad de tener más opciones de centros de atención y especialistas en cáncer. También se encontró que, a mayor capacidad de organizarse, planear actividades y tener actividades sistemáticas, hubo menor percepción de necesidad de cuidado y apoyo, así como de SSI. La relación en el grupo de tratamiento de percibir mayores necesidades psicológicas ante menor fortaleza y confianza propias coincidió 
con lo hallado por Dubey et al. ${ }^{(23)}$. Estos resultados apoyan la noción de que la autopercepción acerca de la capacidad de enfrentar situaciones adversas puede influir en la percepción de necesidad de apoyo externo.

La inexistencia de relación entre NAT y las características sociodemográficas es inconsistente con otras investigaciones que han reportado una relación negativa entre NAT y edad ${ }^{(38-41)}$. Es posible que las correlaciones en los estudios antecedentes (realizados con mujeres mayores) se encuentren dadas por las necesidades de tipo físicas o de cuidado y apoyo que se generaron con el propio envejecimiento, aunado a los efectos a largo plazo de los tratamientos oncológicos ${ }^{(42)}$

La relación positiva entre resiliencia y escolaridad es consistente con lo reportado por estudios antecedentes ${ }^{(26)}$, así como la falta de relación entre edad, estado civil, tiempo desde el diagnóstico y etapa clínica ${ }^{(21,25,43)}$. Estos hallazgos apoyan la noción de que el nivel de resiliencia reportado por las pacientes no se ve influido tanto por experimentar situaciones amenazantes, sino que los recursos proporcionados por la educación escolar les permiten percibir habilidades para relacionarse mejor socialmente y tener mayor confianza en sí mismas ${ }^{(21,26,43-45)}$.

\section{Conclusiones}

Se considera que tanto condiciones externas a la paciente, como propias (psicológicas), pudieron contribuir al hecho de que las pacientes jóvenes percibieran pocas necesidades insatisfechas. Factores que pudieron influir en esta percepción son las condiciones de infraestructura, los servicios de salud proporcionados y el apoyo de la seguridad social, así como las habilidades (auto-confianza, estructura y organización) o recursos (apoyo social y familiar) -resiliencia- percibidos por las pacientes, respectivamente. Sin embargo, para corroborar estos hallazgos será necesario tener estudios con muestras de distintas instituciones de salud mexicanas, de manera que permita conocer si este resultado es generalizable a la población del país.

La consistencia con la literatura internacional en cuanto a que las necesidades de SSI y psicológicas son las más prevalentes, da cuenta de la falta de investigaciones culturalmente sensibles a las preferencias de formatos y fuentes de información, así como de investigaciones que diseñen y evalúen entrenamientos en comunicación médico-paciente.

El resultado de que la etapa de tratamiento y la supervivencia de las mujeres jóvenes inducen necesidades insatisfechas distintas, recalca la importancia de considerar esta información al momento de implementar intervenciones dirigidas tanto a la información como al apoyo psicológico. El hecho que las mayores necesidades percibidas fueran de SSI, confirma la necesidad de estas mujeres jóvenes por mantener un rol activo respecto a la toma de decisiones en la trayectoria de la enfermedad ${ }^{(36)}$.

Estudios futuros podrían ampliar el conocimiento sobre las diferencias en necesidades agregando grupos de control, así como evaluando longitudinalmente la trayectoria de las necesidades a lo largo del proceso de diagnóstico, tratamiento y sobrevivencia del CaMa, de manera que se obtenga un conocimiento más profundo de cada etapa. 
La relación entre percepción de mayores recursos, habilidades propias y los recursos proporcionados por la educación formal reafirma que identificar aquellos componentes de esta última que contribuyan a la percepción de alta resiliencia llevará a implementar, en contextos clínicos, mejores y más adecuadas intervenciones basadas en resiliencia.

Hasta donde conocemos, el presente estudio es el único que ha evaluado NAT y resiliencia en mujeres jóvenes mexicanas con CaMa. Los resultados obtenidos distinguen la importancia de la resiliencia como una variable positiva relacionada con la respuesta emocional, cognitiva y social ante el diagnóstico de CaMa, que es además útil para dar cuenta de la satisfacción de las NAT. A partir de esto, podemos considerar clínicamente significativo desarrollar programas de atención para mujeres jóvenes con CaMa en términos de resiliencia.

\section{Referencias bibliográficas}

1. Knaul F, Nigenda G, Lozano R, Arreola-Ornelas H, Langer A, Frenk J. Breast cancer in Mexico: A pressing priority. Reprod Health Matters 2008; 16:1-11. doi: 10.1016/S09688080(08)32414-8.

2. Villarreal-Garza C, Aguila C, Magallanes-Hoyos MC, Mohar A, Bargalló E, Meneses A, Cazap E, et al. Breast cancer in young women in Latin America: an unmet, growing burden. Oncologist 2013; 18:1298-306. doi: 10.1634/theoncologist.18-S2-26.

3. Villarreal-Garza C, Águila C, Platas A, Lara H. Cáncer de mama en mujeres jóvenes en México: necesidades y retos clínicos. Rev Investig Clínica 2014; 66: 547-558.

4. Adams E, McCann L, Armes J, Richardson A, Stark D, Watson E, Hubbard G. The experiences, needs and concerns of younger women with breast cancer: a metaethnography. Psychooncology 2011; 20: 851-61. doi: 10.1002/pon.1792.

5. Partridge AH, Ruddy KJ. Fertility and adjuvant treatment in young women with breast cancer. Breast 2007;16: Suppl 2: S175-81. doi: 10.1016/j.breast.2007.07.029

6. Bauer KR, Brown M, Cress RD, Parise CA, Caggiano V. Descriptive analysis of estrogen receptor (ER)-negative, progesterone receptor (PR)-negative, and HER2-negative invasive breast cancer, the so called triple-negative phenotype. Cancer 2007; 109: 17218. doi:10.1002/cncr.22618

7. Villarreal-Garza C, Mohar A, Bargallo-Rocha JE, Lasa-Gonsebatt F, Reynoso-Noverón $\mathrm{N}$, Matus-Santos J, et al. Molecular subtypes and prognosis in young mexican women with breast cancer. Clin Breast Cancer 2017; 17: e95-e102. doi: 10.1016/j.clbc.2016.11.007

8. Xiong Q, Valero V, Kau V, Kau SW, Taylor S, Smith TL, et al. Female patients with breast carcinoma age 30 years and younger have a poor prognosis: the M.D (Anderson Cancer Center experience). Cancer 2001;92:2523-8.

9. Durkin K. Developmental Social psychology—from infancy to old age. 3rd ed. Blackwell: Oxford, 2001.

10. Fitch MI. Supportive care framework. Can Oncol Nurs J 2008; 18: 6-14. doi: $10.5737 / 1181912 \times 181614$

11. Ali A., Warner E. Pynk: Breast cancer program for young women. Current Oncol 2013; 20: 34. doi: 10.3747/co.20.1131. 
12. Fitch MI, Gray RE, Godel R, Labrecque M. Young women's experiences with breast cancer: An imperative for tailored information and support. Can Oncol Nurs J 2008; 18: 74-86. doi:10.5737/1181912x1827479

13. Pedersen AE, Hack TF, McClement SE, Taylor J. An exploration of the patient navigator role: perspectives of younger women with breast cancer. Oncol Nurs Forum 2014; 41: 77-88. doi: 10.1188/14.ONF.77-88.

14. Ruddy KJ, Greaney ML, Sprunck-Harrild K, Meyer ME, Emmons KM, Partridge AH. Young women with breast cancer: A focus group study of unmet needs. J Adolesc Young Adult Oncol 2013; 2:153-60. doi: 10.1089/jayao.2013.0014

15. Ruddy KJ, Greaney ML, Sprunck-Harrild K, Meyer ME, Emmons KM, Partridge AH. A qualitative exploration of supports and unmet needs of diverse young women with breast cancer. J Community Support Oncol 2015;13:323-9. doi: 10.12788/jcso.0169.

16. Schumacher JR, Palta M, Loconte NK, Trentham-Dietz A, Witt WP, Heidrich SM, et al. Characterizing the psychological distress response before and after a cancer diagnosis. $\mathrm{J}$ Behav Med 2013;36:591-600. doi:10.1007/s10865-012-9453-x

17. Amar-Amar J, Martinez-Gonzalez M, Utria-Utria L. Nuevo abordaje de la salud considerando la resiliencia. Salud Uninorte. Col. Barranquilla 2013; 29: 124-33.

18. Luthar SS. Resilience in development: a synthesis of research across fi ve decades. In: Cicchetti D, Cohen DJ, editors. Developmental psychopathology. 2nd edn. Hoboken, NJ: Wiley, 2006.p. 739-95.

19. Lim JW, Shon EJ, Paek M, Daly B. The dyadic effects of coping and resilience on psychological distress for cancer survivor couples. Support Care Cancer 2014; 22: 320917. doi: 10.1007/ s00520-014-2334-9.

20. Loprinzi CE, Prasad K, Schroeder DR, Sood A. Stress Management and Resilience Training (SMART) program to decrease stress and enhance resilience among breast cancer survivors: A pilot randomized clinical trial. Clin Breast Cancer 2011; 11:364-8.

21. Schrier M, Amital D, Arnson Y, Rubinow A, Altaman A, Nissenabaum B, et al. Association of fibromyalgia characteristics in patients with non-metastatic breast cancer and the protective role of resilience. Rheumatol Int 2011; 32: 3017-23. doi:10.1007/s00296-0112104-7

22. Brix C, Schleussner C, Fuller J, Roehrig B, Wendt TG, Strauss B. The need for psychosocial support and its determinants in a sample of patients undergoing radiooncological treatment of cancer. J Psychosom Res 2008; 65:541-8. doi: 10.1016/j. jpsychores.2008.05.010.

23. Dubey C, De Maria J, Hoeppli C, Betticher DC, Eicher M. Resilience and unmet supportive care needs in patients with cancer during early treatment: A descriptive study. Eur J Oncol Nurs 2015; 19: 582-8. doi: 10.1016/j.ejon.2015.03.004

24. Guil R, Zayas A, Gil-Olarte P, Guerrero, C, González S, Mestre JM. Bienestar psicológico, optimismo y resiliencia en mujeres con cáncer de mama. Psicooncología 2016; 13: 127 38. doi:10.5209/rev_PSIC.2016.v13.n1.52492

25. Ristevska-Dimitrovska G, Filov I, Rajchanovska D, Stefanovski P, Dejanova B. Resilience and quality of life in breast cancer patients. Open Access Maced J Med Sci 2015; 3: 727 31. doi: 10.3889/oamjms.2015.128

26. Wu Z, Liu Y, Li X, Li X. Resilience and associated factors among Mainland Chinese women newly diagnosed with breast cancer. PLoS One 2016; 11: e0167976. doi: 10.1371/ journal.pone.0167976. 
27. Boyes A, Girgis A, Zucca A, Lecathelinais C. Brief assessment of adult cancer patients perceived needs: Developmental and validation of the 34- item supportive care needs survey (SCNS-SF34). J Eval Clin Pract 2009; 15: 602-6

28. Gálvez-Hernandez CL, Ortega-Mondragón A, Romo-González AG, Mohar-Betancour A, Boyes A, Oñate-Ocaña L, et al. Validation of the Supportive Care Needs Survey (SCNSSF34) for Mexican breast cancer patients [en prensa].

29. Palomar-Lever J, Gómez-Valdez NE. Desarrollo de una escala de medición de la resiliencia con mexicanos (RESI-M). Interdisciplinaria 2010; 27: 7-22

30. Doubova SV, Aguirre-Hernandez R, Gutierrez-de la Barrera M, Infante-Castañeda C, Pérez-Cuevas R. Supportive care needs of Mexican adult cancer patients: validation of the Mexican version of the Short-Form Supportive Care Needs Questionnaire (SCNSSFM). Support Care Cancer 2015; 23: 2711-9. doi: 10.1007/s00520-015-2634-8

31. Avis NE, Crawford S, Manuel J. Psychosocial problems among younger women with breast cancer. Psychooncology 2004; 13: 295-308. doi:10.1002/pon.744

32. Connell S, Patterson C, Newman B. Issues and concerns of young Australian women with breast cancer. Support Care Cancer 2006; 14:419-26. doi:10.1007/s00520-005-0003-8

33. Mohar A, Reynoso N, Villarreal-Garza C, Bargalló-Rocha JE, Arce-Salinas C, LaraMedina F. Cáncer de mama en el Instituto Nacional de Cancerología. Experiencia del seguro popular 2007-2013. Rev Mex Mastol 2015; 5: 6-11.

34. Goss C, Deledda G, Bottacini A, Chiodera F, Mazzi MA, Ballarin M, et al. Information needs of female Italian breast cancer patients during their first oncological consultation. Eur J Oncol Nurs 2015; 19:451-7 doi: 10.1016/j.ejon.2015.02.003

35. Sutliff M, Walker D, Esser M, Rowe J, Lewis S, McCann M. Information needs of young women with metastatic breast cancer to manage their treatment, side effects and clinical trials. Breast 2015; 24: S37-S37.

36. Thewes B, Meiser B, Rickard J, Friedlander M. The fertility-and menopause-related information needs of younger women with a diagnosis of breast cancer: a qualitative study. Psychooncology, 2003; 12: 500-11. doi. 10.1002/pon.685

37. McLachlan K. Information and support needs of young women with breast cancer. Cancer Nurs Pract 2009; 8: 21-4.

38. Abdollahzadeh F, Moradi N, Pakpour V, Rahmani A, Zamanzadeh V, Mohammadpoorasl A, et al.Un-met supportive care needs of Iranian breast cancer patients. Asian Pac J Cancer Prev 2014; 15: 3933-8. doi: 10.7314/APJCP.2014.15.9.3933

39. Brédart A, Kop JL, Griesser AC, Zaman K, Panes-Ruedin B, Jeanneret W, et al. Validation of the 34-item Supportive Care Needs Survey and 8-item Breast module French versions (SCNS-SF34-Fr and SCNS-BR8-Fr) in breast cancer patients. Eur J Cancer Care 2012; 21: 450-9. doi: 10.1111/j.1365-2354.2012.01356.x.

40. Liao M-N, Chen S-C, Chen S-C, Lin Y-C, Hsu Y-H, Hung HC, et al. Changes and predictors of unmet needs in Taiwanese women with newly diagnosed breast cancer. Oncol Nurs Forum 2012; 39: E380-89. doi: 10.1188/12.ONF.E380-E389.

41. Li WWY, Lam WWT, Au AHY, Ye M, Law WL, Poon J, et al. Interpreting differences in patterns of supportive care needs between patients with breast cancer and patients with colorectal cancer. Psychooncology 2012; 22: 792-8. doi: 10.1002/pon.3068.

42. Ha ̈rtl K, Engel J, Herschbach P, Reinecker H, Sommer H, Friese K. Personality traits and psychosocial stress: quality of life over 2 years following breast cancer diagnosis and psychological impact factors. Psychooncology 2010; 9:160-9. doi: 10.1002/ pon. 1536 
43. Scali J., Gandubert C, Ritchie K, Soulier M, Ancelin ML, Chaudieu I. Measuring resilience in adult women using the 10-items Connor-Davidson Resilience Scale (CDRISC). Role of trauma exposure and anxiety disorders. PloS one 2012; 7: e39879. doi: 10.1371/journal.pone.0039879.

44. Crespo-Fernández L, Rivera-García M. El poder de la resiliencia generado por el cáncer de mama en mujeres en Puerto Rico. Rev Puertorriqueña Psic 2012; 23: 109-126.

45. García S, López M, Latorre J. Ansiedad, resiliencia e inteligencia emocional percibida en grupo de mujeres con cáncer de mama. Ansiedad Estrés 2015; 21:115-25 